\title{
Pediatric insomnia: new insights in clinical assessment and treatment options
}

\author{
O. BRUNI', M. ANGRIMAN² \\ ' Department of Developmental and Social Psychology, Sapienza University; \\ 2 Department of Pediatrics, Child Neurology and Neurorehabilitation Unit, Central Hospital of Bolzano
}

\section{A B S T R A C T}

Sleep disorders in children can compromise quality of life of both children and families and it is known that chronic sleep deprivations is associated with poorer developmental outcome, overweight and behavioral disturbances. Clinicians should incorporate questions about sleep into their routine health assessment, and the assessment of insomnia should follow a medical approach. Primary and secondary contributing factors should be assessed, as well as maladaptive behaviors related to sleep.

A careful examination of sleep/wake schedule, abnormal movements or behavior during sleep, and daytime consequences of sleep disruption or deprivation is mandatory.

Sleeping environment, and bedtime routines should be examined to identify behavioral issues related to sleep.

Polysomnography is not routinely indicated for children with insomnia, but actigraphy can give an objective estimation of sleep parameters.

The Authors propose a new classification of pediatric insomnia, based on both genetic and clinical aspects, and suggest specific treatment options, including sleep hygiene, behavioral strategies and pharmacological treatment.

Key words

Pediatric insomnia $\bullet$ Pharmacological treatment $\bullet$ Behavioral treatment

\section{Introduction}

Identification of sleep problems in children is important because a growing body of evidence suggests a link between sleep disorders and physical, cognitive, emotional, and social development. Children with neurodevelopmental problems, learning difficulties, or behavioral disturbances may be at heightened risk for sleep problems compared with the general pediatric population (Mindell, 2010).

\section{Prevalence of Pediatric insomnia}

The prevalence of pediatric insomnia ranges from 10 to $20-30$ percent among general pediatric populations (mainly based on the first 5 years of life); this variation is related to study methodology, different definition of insomnia and inclusion criteria (i.e. parental report of problematic sleep or definition of insomnia as more than 3 awakenings per night, etc.), with a much higher prevalence in children with neurodevelopmental and chronic medical and psychiatric conditions (Owens et al. 2011, Angriman et al., 2015, Honaker and Meltzer, 2014). Moreover, insufficient sleep constitutes a major concern in adolescence, with the majority of US adolescents sleeping less than recommended on school nights. (Owens, 2014).

The definition and clinical presentation vary with age, developmental status, and culture. An interesting cross-cultural study on normal sleep in children aged 0-36 months evaluated through the Brief Infant Sleep Questionnaire filled out by parents/caregivers, reported a different prevalence of sleep problems 
ranging from $10 \%$ in Vietnam and Thailand, to $25 \%$ to $30 \%$ in the United States and Australia and even $75 \%$ in China and Taiwan (Mindell et al., 2010)

For parents of infants and toddlers night wakings are one of the most common sleep complaints, with $25 \%$ to $50 \%$ of children older than 6 months of age continuing to awaken during the night. Bedtime resistance is found in $10 \%$ to $15 \%$ of toddlers. Difficulties falling asleep and night wakings (15\%$30 \%$ ) are also common in preschoolers. In older children the main insomnia symptoms reported are sleep onset difficulty $(15 \%)$ and sleep-related anxiety (11\%). (Owens and Mindell 2011)

The presentation, natural history, and response to treatment may differ considerably between adults and children and even within the pediatric age group, the clinical manifestations of sleep problems may vary by age and developmental level.

Classification of Pediatric Insomnia in the International Classification of Sleep Disorders

According to the previous International Classification of Sleep Disorders, ICSD-2 (American Academy of Sleep Medicine, 2005), "pediatric insomnia" was defined as a "repeated difficulty with sleep initiation, duration, consolidation, or quality that occurs despite age-appropriate time and opportunity for sleep and results in daytime functional impairment for the child and/or family."

The diagnosis of behavioral insomnia of childhood (BIC) was introduced in 2005 in ICSD-2 as a unique diagnostic entity to emphasize sleep difficulties that result from inappropriate sleep associations or inadequate parental limit setting. The diagnosis of sleep-onset behavioral insomnia was characterized by reliance on maladaptive and inappropriate sleep associations such as rocking, watching television, falling asleep in the parents' bed, and so forth. The child is usually unable to fall asleep in the absence of these conditions at both bedtime and following nocturnal arousals. Inadequate parental limit setting can also result in a form of behavioral insomnia characterized by sleep-onset delay secondary to a child's refusing to go to bed or stalling.

The ICSD third edition (ICSD-3; American Academy of Sleep Medicine, 2014) describes Chronic Insomnia disorder with no specific pediatric classification; as a consequence, a related unresolved issue is whether the current global classification promotes a generic approach to insomnia therapy that ultimately fails to benefit some insomnia subgroups. The ICSD-3 defines chronic insomnia as "a persistent difficulty with sleep initiation, duration, consolidation, or quality that occurs despite adequate opportunity and circumstances for sleep, and results in some form of daytime impairment". Based on the ICSD-3, there are several issues that should be considered when approaching an infant/ child with insomnia:

1) parents may have unrealistic sleep expectations for their children and predispose them to insomnia by putting them in bed too early or assigning them too much time in bed each night.

2) child insomnia is often comorbid with behavioral disturbances, such as irritability, hyperactivity or attention difficulties, or other comorbid medical and psychiatric conditions.

3) social risk factors like difficulty home situations, safety concerns, caregiver relationship and domestic abuse, should be considered or excluded

4) if the children had a current or past history of medical problems, parents may have difficulty setting limits, because of guilt, a sense that the child is "vulnerable," or concerns about doing psychological harm.

5) environmental factors such as the child sharing a room with others and cramped living accommodations may contribute to negative sleep-onset associations or poor limit setting

6) the psychological asset of the parents (especially depressive symptoms) should be always evaluated A debated issue is at what age the diagnosis of insomnia could be done: because children are not expected to sleep through the night with regularity until they are 3-6 months of age, 6 months is a reasonable age to first consider a diagnosis of chronic insomnia disorder, unless the sleeplessness is very marked at an earlier age.

Classification of Pediatric Insomnia in the Diagnostic and Statistical Manual of Mental Disorders

In the Diagnostic and Statistical Manual of Mental Disorders, $5^{\text {th }}$ edition, DSM-5 (American Psychiatric Association, 2013) there is no classification of sleep disorders specific for childhood.

The DSM-V includes sleep disorders defined according to criteria that are common for children and 
adults, although, in some instances, developmental features of particular sleep disorders are specified. The DSM-5 integrated pediatric and developmental criteria and also replaced "primary insomnia" with the diagnosis of "insomnia disorder", a switch to avoid the primary/secondary designation when this disorder co-occurs with other conditions and to reflect changes throughout the classification. Furthermore, it introduced a temporal criterion (more than 3 "bad nights" at week for the last 3 months). DSM-5 underscores the need for independent clinical attention of a sleep disorder regardless of mental or other medical problems that may be present.

\section{Clinical approach to pediatric insomnia}

Insomnia in children and adolescents can be caused or related to medical (allergy, gastroesophageal reflux, medication, pain) and behavioral (irregular sleep schedule or negative sleep-onset associations) disorders.

The manifestations of childhood insomnia are mainly represented by bedtime struggles, difficulty falling asleep, and/or frequent or prolonged night wakings requiring parental intervention in the first 3 years of life; by bedtime refusal or fear to go to sleep in school-age children and by delayed sleep phase and/or difficulty initiating sleep in adolescence.

The assessment of sleep and sleep disturbances in children is performed trough subjective (i.e., information reported by the child and/or parents, questionnaires) or objective tools (i.e., measure of motor or neurophysiologic parameters).

\section{Open questions on definition, classification and treatment of pediatric insomnia}

Although several studies have been published on the treatment of insomnia in infants and children the translation of research findings to practice settings remains unclear for several reasons:

a) most treatment studies define sleep problems by symptoms and fail to classify using diagnostic criteria (Mindell et al., 2006);

b) there is no indication on the appropriate treatment for specific insomnia subtypes (Morgenthaler et al., 2006); c) no clear evidence on non-pharmacological and pharmacological treatment frequency and duration (Owens et al., 2005)

d) the frequent clinician follow-up (e.g., daily phone calls) might be not practical;

e) few data are available on treatment of older children and adolescents. (Meltzer \& Mindell, 2014);

f) no specific instruments for assessing the severity of insomnia are available;

g) there is a worldwide trend toward increased use of medication to treat pediatric insomnia despite a lack of efficacy data (Mindell, et al., 2006).

\section{Proposal of a clinical classification of pediatric insomnia}

The generic classification of ICSD-3 and DSM-5 of insomnia necessarily does not allow an accurate clinical assessment of insomnia and could be misleading for finding the correct patient-oriented treatment approach. The clinician's challenge is to elucidate the possible causative factors and find the correct therapy either non-pharmacological or pharmacological.

For these reasons we propose a new clinical categorization of childhood insomnia that might be conceptualized as follows (Bruni et al., 2013):

- Insomnia with motor hyperactivity

- Insomnia with prevalent middle of the night awakenings

- Insomnia with multiple night awakenings and falling asleep difficulties

These three different types might underlie different pathophysiological mechanisms:

a) the insomnia characterized by motor hyperactivity (parental report of a child that kick the legs or described as a "horse in the bed") is probably linked to a dopaminergic dysfunction since this resemble the cases of Restless Legs syndrome reported by Picchietti et al (2008) that in early infancy showed chronic sleep-onset problems and sleep-maintenance problems. In a recent study it has been shown that symptoms of restless leg syndrome may already start in the first year of life and are related to low serum ferritin level. The Authors showed that the most striking single symptom was awakening after 1-3 $\mathrm{h}$ of sleep followed by screaming, crying, kicking and slap- 
ping the legs or by verbally expressing that the legs 'hurts' with a seemingly comforting effect of massage and cycling movements performed by the parents (Tilma et al., 2013). Recently we described the case of a toddler with severe insomnia, bedtime and nocturnal hyperactivity, night awakenings associated with leg kicking and rubbing, responsive to gabapentin and highly suggestive of restless legs syndrome but presenting as severe insomnia. (Bruni et al, in press).

b) the insomnia with prevalent middle of the night awakenings could resemble the insomnia of depressed people characterized by no falling asleep troubles but prolonged midnight awakening with difficulty returning to sleep. Recent studies have demonstrated a link between sleep difficulties in childhood and depression in mid-adolescence (Gregory et al., 2005) and in adulthood (Green et al., 2015). It has been shown that some antidepressants could improve sleep quality: administration of 5HT2A receptor antagonists determined an increase of Slow Wave Sleep (SWS) and a reduction of Rapid Eye Movement (REM) sleep and an improvement of sleep continuity (Monti, 2011)

c) the insomnia with multiple night awakenings and falling asleep difficulties is often a symptom related to infants who present with milk allergy or gastroesophageal reflux and therefore are highly suspected to be related to a histaminergic dysfunction. The histaminergic system in the brain is exclusively localized within the posterior hypothalamus with projection to almost all the major regions of the central nervous system. Administration of histamine or $\mathrm{H} 1$ receptor agonists induces wakefulness, whereas administration of $\mathrm{H} 1$ receptor antagonists promotes sleep. The first generation of antihistamines easily penetrate the blood brain barrier and cause drowsiness and sedation. Several of these antihistamines including the non-selective $\mathrm{H} 1$ receptor antagonists from the phenothiazine class and "over the counter" diphenhydramine, have positive effects on daytime sleepiness as well as on subjective and objective measures of nocturnal sleep in healthy human subjects (Thakkar, 2011). This kind of categorization obviously has important therapeutical implications and allows the clinician to personalize pharmacological treatment based on the hypothesized neurotransmitter dysfunction.
In order to correctly categorize the specific type of insomnia a careful family and personal history should be collected in order to evaluate the presence of symptoms/diseases that could be associated with the hypothesized categorization of insomnia.

This approach is of high relevance since recent studies emphasized the role of genetic influences on the development of insomnia of childhood suggesting that the influence of genetic factors should not be underestimated and could contribute to selecting appropriate personalized treatment.

For example, a study showed that heritability contributed for $30.8 \%$ on nocturnal sleep duration, for $36.3 \%$ on diurnal sleep duration and for $35.3 \%$ on night wakings (Brescianini et al. 2011); moreover, Touchette et al (2013) observed that variance in consolidated nighttime sleep duration was largely influenced by genetic factors with a critical environmental timewindow influence at $\sim 18$ months. A strong heritability $(71 \%)$ was observed for the short-persistent $(<10$ hours) nighttime sleep duration trajectory and by contrast, the increasing nighttime sleep duration trajectory ( $>11 \mathrm{hs)}$ ) did not appear highly heritable $(58 \%)$. All these evidences pointed out that we need to obtain a very careful family history to understand the role of genetic influences on sleep and choose the appropriate approach. LeBlanc et al. (2009) point out that family history was the second strongest predictive factor in new cases of insomnia syndrome with the implication that there may be a familial predisposition and in other words, a vulnerable phenotype. Further, there was a trend towards a higher familial incidence in those reporting earlier onset vs. those reporting a later onset. Moreover, evidence suggests that the expression of 5HTTLPR, which affects synaptic serotonin levels, is critical in the development of the neonatal brain and also the 5HTTLPR contributes to the onset of insomnia rather than the severity (Harvey et al., 2014).

In summary, family and twin studies highlight that certain genetic predisposing factors of individual vulnerability might contribute ting to the onset of insomnia. However, also several epigenetic mechanisms seem to be involved in the regulation of sleep and in the development of insomnia: stressful experiences during prenatal/early life development may contribute to changes in stress reactivity that may persist into adulthood. If epigenetic mechanisms are potentially reversible via environmental or pharmacological 
interventions it might be hypothesized that both cognitive behavioral treatment for insomnia or pharmacological interventions might influence epigenetic modification in insomnia (Palagini et al., 2014).

From the previous studies it is clear that a predisposition to insomnia seems to exist, driven by factors associated with response to stress at both a psychological and physiological level. Understanding vulnerability to insomnia will inform about the aetiology of other disorders, like depression or anxiety. Therefore, in order to summarize our hypothesis on the different types of insomnia we propose the following treatment-scheme:

a) An infant who presents with no particular difficulties in falling asleep but prolonged middle-ofthe-night awakenings and a family and clinical history of insomnia, parasomnias, headache/ migraine, depression and mood disorders probably underlie a serotonergic dysfunction and therefore should be treated with serotonergic drugs. Obviously selective serotonin reuptake inhibitors (SSRI) are not indicated in infants and children and therefore L-5-hydroxytryptophan (precursor of serotonin) could be the choice for this type of insomnia; it does not have opioid-like effects and does not limit cognitive performance or inhibit arousal from sleep.

b) An infant presenting with difficulty in falling asleep linked to restless legs or kicking legs and with nocturnal hyperactivity and a family and clinical history of restless legs syndrome or periodic limb movements during sleep, iron deficient anemia, growing pains might indicate a dopaminergic dysfunction and should be evaluated for anemia and eventually treated with iron.

c) An infant showing multiple night awakenings and falling asleep difficulties with a clinical history of atopic dermatitis or milk allergy or gastroesophageal reflux and with a high presence of allergies in the family might reveal a histaminergic dysfunction. In this case obviously the treatment of choice should be the first generation of antihistamines with high affinity for the $\mathrm{H} 1$ receptor.

\section{Evaluation of pediatric insomnia}

a careful sleep and medical history and the use of a sleep diary, guided by an understanding of normal sleep physiology, provides the foundation for sorting out the cause of a sleep disorder and guide his management.

Chronic conditions such as reactive airways disease, gastroesophageal reflux, and causes of chronic pain should be excluded; neurodevelopmental disabilities [i.e. cerebral palsy, intellectual disability, autism, blindness, Attention Deficit/Hyperactivity Disorders (ADHD)] are associated with elevated risk for sleep disorders and should be assessed.

A thorough medication history related to specific pharmacological treatments for different medical/ neurological/psychiatric disorders and/or sleep disturbances should be collected.

The clinician should evaluate the sleep/wake schedule, if the prevalent difficulties is in initiating or in maintaining sleep, abnormal movements or behavior during sleep, and daytime symptoms (eg, sleepiness, inattentiveness, or irritability). The history should include details about the duration and frequency of the problem, temporal profile of onset (abrupt, gradual, intermittent), and degree of variability from night to night. The clinician should note what interventions or strategies have been tried and whether medications have been used.

As an example, a quick subjective instrument to assess sleep is known as BEARS (B = Bedtime issues, $\mathrm{E}=$ Excessive daytime sleepiness, $\mathrm{A}=$ night Awakenings, $\mathrm{R}=$ Regularity and duration of sleep, $\mathrm{S}=$ Snoring) (Mindell et al., 2010). The clinician should keep in mind that the child with excessive sleepiness may exhibit motor overactivity, inattentiveness, irritability, or oppositional behavior, other than classical signs of sleepiness of the adult patients.

Structured questionnaires, such as the Sleep Disturbance Scale for Children (Bruni et al., 1996) may help in data collection.

Sleep log: In order to assess sleep patterns over time, the child and/or his parents can fill out a sleep diary, describing bedtime, and wake up time, sleep onset, the frequency and duration of nighttime awakening and naps duration. Completion of a sleep log during the two weeks prior to evaluation may provide important information regarding the sleep/wake pattern and nocturnal events.

Physical examination: Developmental milestones and growth parameters should be evaluated. Examination of the oropharynx is mandatory to 
reveal evidence of tonsillar or adenoidal hypertrophy, mandibular hypoplasia, retrognathia as observation of mouth breathing or noisy breathing. Neurological examination should be useful to evaluate problems related to psychomotor retardation

Actigraphy: It consists in a wrist-watch-like device (put on the wrist or ankle) that continually records movements and monitors cycles of physical activity and rest. Objective sleep parameters (sleep/wake periods, total duration of sleep, number of awakenings, and length of sleep onset) are inferred by the patterns of rest/movement.

Polysomnography: Polysomnography (PSG) typically consists of an all-night recording performed in the sleep laboratory. A number of physiological parameters are measured, including EEG, eye movements, muscle tone, respiratory function (including air flow at the nose and mouth, respiratory movements of the chest and abdomen, and oximetry), electrocardiogram (EKG), limb movements, snoring or vocalizations, and video tape to characterize movements during sleep.

Polysomnography is not routinely indicated for evaluation of difficulty initiating or maintaining sleep but is useful to exclude other sleep disorders.

\section{Fundamentals of treatment}

Good sleep practices and behavioral interventions are the first recommended treatments for pediatric insomnia (Honaker et al. 2014). It is important to discuss parents' knowledge and beliefs as well as strategies they have used to help address their child's sleep problems.

\section{Sleep hygiene}

Healthy sleep practices include daytime and nighttime sleep practices that positively impact sleep initiation/maintenance, sleep quantity and quality; it usually includes bedtime routine, consistent bedtime and wake time, a quiet, dark and cool bedroom, avoidance of caffeinated products, and daily physical activity. A critical aspect of sleep hygiene is the use of technology in the bedroom (computer, TV, cell phone, video games), which is clearly associated with decreased sleep quantity and quality in children (Mindell et al. 2009).

\section{Behavioral strategies}

Behavioral treatments for sleep difficulties are commonly considered as brief and highly effective, with many options available.

Several behavioral techniques are available, and the clinician should propose to the family the most appropriate, based on parental preferences and child temperament (Meltzer, 2010):

Unmodified Extinction. The child is placed in bed while awake, left alone until asleep, and night wakings are ignored. The infant learns to selfsoothe once realizing that nighttime crying does not result in parental attention. Parents ignore all child behaviors and requests at bedtime and the middle of the night including crying, calling out, and more extreme behaviors such as throwing objects or vomiting. When used consistently, extinction can over the course of 3 to 5 days eliminate these child behaviors, as the child no longer depends on the parents to be present at bedtime because she or he develops new associations or self-soothing skills. This method is generally very effective but many parents disagree with this approach. (Reid et al 2009).

Graduate extinction. This involves ignoring negative behaviors (i.e., crying) for a given amount of time before checking on the child. The parent gradually increases the amount of time between crying and parental response. The parent remains in the room during extinction, acting as a reassurance for the child but providing little interaction

This technique is easier for parents to implement and to accept because it involves more flexibility for parents to pace the intervention, and typically less child distress; however, it can take longer time to resolve the problem. Any combination of parent behaviors can be gradually modified, such as physical contact with the child (eg, breast feeding, holding, patting.), verbal responses, proximity to the child, and frequency and duration of check-ins. (Reid et al 1999). Sleep scheduling/Bedtime fading. These techniques involve delaying bedtime closer to the child's target bedtime. The goal of this treatment is for the child to develop a positive association between being in bed and falling asleep rapidly. Bedtimes can be gradually moved earlier.

Modification of the child's schedule is needed when the child's bedtime is exceedingly early or late, or if the timing of naps interferes with sleep onset 
at bedtime. Schedule problems can arise when a child's optimal sleep time (controlled by their intrinsic circadian phase) conflicts with parental or other environmental demands.

For both preschool and school-age children, shifting to a later bedtime may increase sleep propensity and facilitate a rapid sleep onset. However, a late bedtime can result in the child been overly tired, behaviorally dysregulated, and hyperaroused. There is a naturally occurring increase in arousal level that occurs within an hour of the optimal bedtime, and education about this burst of activity is important, as it can be a point of conflict resulting in increases in parents' and children's arousal levels and frustration. Ignoring, or shifting the child's focus to an engaging, but quiet activity can be helpful. In general, when combined with a positive routine and after the child learns to go to sleep more quickly, parents can gradually advance the bedtime with the goal of increasing the child's sleep time. (Reid et al. 2009) Cognitive strategies. These strategies are used to address nonproductive beliefs about sleep, including the belief that the child cannot change his/her sleep difficulty. They have been mainly used with older children and adolescents (Bootzin \& Stevens 2005). Coping strategies..Diaphragmatic breathing and progressive muscle relaxation can be introduced to mediate somatic tension at sleep onset. It may be useful to record diaphragmatic breathing and progressive muscle relaxation exercises at the consultation and issuing detailed instructions to aid practice at home (Morgenthaler et al. 2006).

All these techniques can be combined and integrated to better adapt to the child and the family. An example of an integrated approach is reported in Table I.

\section{Evidence of efficacy of behavioral treatment}

A recent meta-analysis of 16 controlled trials on behavioral treatments for insomnia in children and qualitative analysis of 12 within-subject studies (total $n=2,560$ ) reported significant effects for sleep-onset latency, number of night wakings, and duration of night wakings, and sleep efficiency, with small to large effect sizes across the controlled clinical trials involving typical children. No significant effects were found for the two studies conducted with special needs populations. The conclusion of this study showed that moderate-level evidence sup- ports behavioral interventions for pediatric insomnia in young children and even low evidence for children, adolescents, and those with special needs (Meltzer and Mindell 2014).

Following the results of this study we should reconsider the claimed "efficacy" of behavioral interventions; more studies are needed to help identify factors that may predict treatment success and to tailor behavioral interventions for young children based on child (e.g., temperament, age), parent, and environmental factors (Meltzer, 2010). Finally, more longitudinal studies are needed to demonstrate whether treatment benefits for insomnia are maintained over time and to examine other functional outcomes (child mood, behavior, health, as well as parental mood, marital satisfaction, and family functioning).

\section{Pharmacological treatment}

Children who do not respond to behavioral interventions should be considered for pharmacological management of insomnia. Currently, there are no U.S. Food and Drug Administration (FDA)approved medications for the treatment of insomnia in children and pharmacological treatment should always be considered in combination with behavioral treatment. (Angriman et al., 2015; Pelayo and Yuen 2012).

It should be taken into account that drugs could be initially useful for parent and child's relief and in general it is better not to wait a long time to treat insomnia; it is better to implement a brief drug trial than act later on a chronic insomnia. Also when a drug has been administered, abrupt discontinuation should be avoided and the treatment should be carefully monitored since there is a natural inclination of the parents to give the lowest dose (Pelayo and Dubik 2008). Finally it should be reminded that cognitive-behavioral therapy should always be associated to drug treatment to ensure the best long term efficacy (Owens et al., 2005).

It is of interest that about $50-60 \%$ of pediatricians use drugs for insomnia in infants and children (Bruni et al., 2004; Owens et al., 2009; Huessler et al., 2013) but, despite the widespread use of prescription therapies such as clonidine, antidepressants, mood stabilizers, and antihistamines, little data exist on 
Table I. - Integrated behavioral approach for insomnia of childhood.

1. Create solid and positive bedtime routines (i.e. songs, books, relaxing activities.)

2. If possible, put the child in bed sleepy but not fully asleep.

3. Put in the child's bed only few familial objects he can use to sooth himself in the case of nocturnal awakenings (avoid plushes or dangerous objects)

4. Establish a constant "good-bye phrase" for example: "You can sleep alone here with your favorite toys".

5. Before leave the child's room, give a plausible explanation ("mama goes to the kitchen to drink some water and then come back to you")

6. Speak to the child from the other room to reassure him.

7. If he begins to cry, let him cry for a brief period (5-10 seconds) before returning in the room

8. Reassure the child letting him in his bed and remain in the room until he has calmed down; reduce as much as possibile the direct interaction with the child

9. Left the room repeating point 4

10. If the child cries, return in the room and repeat from point 7 awaiting a little more time (10-15 seconds) and then point 8 and 9

11. Next night repeat from point 1 to 10 , increasing time of awaiting of 10 seconds

For waiting times in case of nocturnal awakenings, follow these indications:

\begin{tabular}{|l|c|c|c|}
\hline & $\mathbf{1}^{\circ}$ awakening & $2^{\circ}$ awakening & $3^{\circ}$ awakening \\
\hline Day 1 & 10 seconds & 15 seconds & 30 seconds \\
\hline Day 2 & 20 seconds & 25 seconds & 40 seconds \\
\hline Day 3 & 30 seconds & 35 seconds & 50 seconds \\
\hline Day 4 & 40 seconds & 45 seconds & 60 seconds \\
\hline Day 5 & 50 seconds & 55 seconds & 70 seconds \\
\hline Day 6 & 60 seconds & 65 seconds & 80 seconds \\
\hline Day 7 & 70 seconds & 75 seconds & \\
\hline
\end{tabular}

their efficacy for the treatment of insomnia in children and adolescents. (Mindell et al., 2006)

In the following paragraphs a list of the commonly used drugs for insomnia are listed together with the appropriate dosage and side effects.

Antihistamines. In view of their sedative and hypnotic effects, antihistamine are often prescribed for sleep problems in children, however these agents may worsen obstructive sleep apnea (OSA), and also may suppress REM sleep. (Pelayo and Yuen 2012). The first generation of antihistamines bind to $\mathrm{H} 1$ receptors in the CNS and have minimal effects on sleep architecture. They are often the more acceptable choice for many families, commonly well tolerated and may acutely improve sleep and speed up behavioral programs. Common side effects are sedation, paradoxical effects, ataxia and dizziness.

Very few studies have been conducted with antihistamines in children with insomnia reporting conflicting results. Trimeprazine was used in $22 \mathrm{ch}$. with night wakings showing a moderate improvement (France et al., 1999); niaprazine showed a decrease of sleep onset latency and an increase of sleep duration (Ottaviano et al, 1991) even if compared with benzodiazepines (Montanari et al., 1992); diphenhydramine vs. placebo in children with insomnia showed a decrease of sleep latency and night awakenings in one study (Russo et al., 1976) while other studies showed no more effectiveness than placebo (Merenstein et al., 2006; Gringras, 2008)

Iron: Iron is a co-factor for tyrosine hydroxylase the enzyme responsible for catalyzing the conversion of the amino acid L-tyrosine to dopamine.

Children with ADHD and serum ferritin levels $\leq 45 \mu \mathrm{g} / \mathrm{l}$ had significantly higher scores on the subscale 'Sleep wake transition disorders' of the sleep disturbance scale for children compared with children with serum ferritin levels $>45 \mu \mathrm{g} / \mathrm{l}$ (Cortese et al., 2009) suggesting that children with ADHD and sleep disorders, in particular RLS, may be a subgroup particularly at risk 
for iron deficiency and severe ADHD symptoms. The pathophysiology by which abnormal iron metabolism may be associated with RLS and insomnia is not clear. It has been shown that the cerebrospinal fluid concentration of ferritin is reduced while the concentration of transferrin is elevated (Early et al. 2000). Furthermore, plasma iron level demonstrates a circadian rhythm with evening and night values being about half of morning values and this may be related to the observation that RLS symptoms tend to be worse at night determining insomnia (Cortese et al., 2012).

Moreover, iron deficiency anemia was reported to be associated with higher motor activity during sleep, shorter night sleep duration and higher frequency of night waking (Kordas et al., 2008) and supplemental iron was associated with longer sleep duration (Kordas et al., 2009).

Because iron deficiency is common in children, measuring the ferritin level is reasonable. Iron replacement should be initiated if ferritin levels are less than $50 \mathrm{mcg}$ per L, and they should be rechecked in three months (Kryger et al., 2002); although the risk of iron overload is very low, parents should be asked for a personal and family history of hemochromatosis or unexplained liver disease.

L-5-hydroxytryptophan: it is a precursor of serotonine and melatonine, widely used in the 80 s for treatment of sleep disorders and headache prophylaxis. It does not have opioid-like effects and does not limit cognitive performance or inhibit arousal from sleep (Lieberman, 1985).

In the literature several positive effects on sleep are reported: improvement of sleep latency (Korner 1986; Schneider-Helmert and Spinweber, 1986; Hartmann and Spinweber, 1979); increased stage IV sleep noted at even lower doses $250 \mathrm{mg}$ (Hartmann and Spinweber, 1979); evidence of therapeutic effect for sleep terrors in children at dosage of $2 \mathrm{mg}$ / $\mathrm{kg}$ (Bruni et al, 2004)

Melatonin: is a chronobiotic drug crucial for the regulation of the sleep-wake cycle. In older children and adults, its production and secretion begin in the evening and peak during the night between 02:00 and 04:00; its production and release are inhibited by light. MLT is sintethyzed in the pineal gland from based on the following pathway: à 5-hydroxytryptophan à serotonine à $\mathrm{N}$-acetilserotonine à melatonin).
Different studies showed efficacy of MLT (1 to 10 $\mathrm{mg}$ ) in improving sleep in autistic children (Andersen et al., 2008; Giannotti et al., 2006) and in children with neurodevelelopmental disabilities (Wasdell et al., 2008). Further MLT at a dosage of $5 \mathrm{mg}$ was effective in ADHD children with delayed sleep phase syndrome (DSPS) and insomnia (Van Der Heijden et al., 2005 and 2007; Smits et al., 2003; Weiss 2006) In general MLT for treatment of chronic sleep onset insomnia in children is effective in a dosage of $0.05 \mathrm{mg} /$ $\mathrm{kg}$ given at least 1 to $2 \mathrm{~h}$ before desired bedtime (van Geijlswijk et al., 2010). Therefore there is now a greater understanding that low doses $(0.5 \mathrm{mg})$ can be effective for some children, with diminishing benefit with doses exceeding $6 \mathrm{mg}$ and, unlike traditional hypnotics such as chloral hydrate and the benzodiazepines, melatonin does not affect sleep architecture (Bruni et al., 2015)

Clonazepam: Benzodiazepines bind to the benzodiazepine subunit of the gamma aminobutyric acid (GABA) chloride receptor complex, facilitating the action of the inhibitory neurotransmitter GABA. These hypnotics have long been the first choice treatment for insomnia in adults, but raise concerns about cognitive impairment, rebound insomnia, and the potential risk for dependence. These concerns and little evidence-based data availability in the pediatric population, contribute to limit their use in children. (Kotagal, 2012).

Clonidine: Clonidine is an antihypertensive drug with sedating properties; it is a central $\alpha 2$ agonist that decreases adrenergic tone but mechanism of sedation is not clear.

It is rapidly absorbed, onset within $1 \mathrm{~h}$, and peak effects in 2-4 h. Starting dose is usually $50 \mu \mathrm{g}$, increased in $50 \mu \mathrm{g}$ increments. Side effects are represented by hypotension, bradycardia, anticholinergic effects, irritability, dysphoria, tolerance. (Owens, 2009)

No randomized trials of clonidine specifically for children with insomnia exists but the few studies showed a certain efficacy on sleep latency and night wakings. Side effects include hypotension, bradycardia, irritability, anticholinergic effects (e.g., dry mouth), and REM suppression. (Nguyen et al., 2014)

Vitamin D: Clinical research on the relation between vitamin $\mathrm{D}$ and sleep is ongoing, and few studies have been published on the role of vitamin $\mathrm{D}$ metabolism 
and sleep disorders. Preliminary data suggest the possibility that altered vitamin D metabolism could play an important role in the presentation and severity of sleep disorders (McCarty et al., 2014). Vitamin D is related do dopamine metabolism, so it could be useful to investigate vitamin levels in association with iron parameters in children with motor hyperactivity during sleep.

Zolpidem: There are very few studies conducted in children. A study on 6-11 years or 12-17 years children with ADHD and insomnia received treatment with zolpidem at $0.25 \mathrm{mg} / \mathrm{kg}$ per day (max $10 \mathrm{mg}$ / day) vs. placebo. Mean change in latency to persistent sleep at week 4 did not differ between zolpidem and placebo groups, although subjective data tended to indicate an overall improvement in insomnia for the older age group based on Clinical Global Impression scores following treatment with the active drug. No next-day residual effects of treatment and no rebound phenomena occurred after treatment discontinuation. Most-frequent adverse events ( $>5 \%)$ were dizziness, headache, and hallucinations (Blumer et al., 2009)

\section{Conclusions}

Insomnia in children has multifactorial origin and can cause impairment in quality of life of both patients and families.

The medical approach should follow the pathway of sleep medicine, examining medical and genetic contributing factors to find a patient-oriented treatment approach. Behavioral treatment strategies, and pharmacological options are available. Despite the widespread use of pharmacological treatment, the lack of well designed, controlled studies concerning the efficacy, tolerability, dosage, and safety profile of hypnotic medications in children raise the need of further research in this field of sleep medicine.

\section{References}

American Academy of Sleep Medicine International Classification of Sleep Disorders, 2nd ed.: Diagnostic and coding manual. 2005, Westchester IL: AASM.

American Academy of Sleep Medicine International Classification of Sleep Disorders, 3nd ed. 2014, Darien IL: AASM.
American Psychiatric Association Diagnostic and Statistical Manual of Mental Disorders, Fifth Edition, DSM-5. 2013, Arlington, VA: American Psychiatric Publishing.

Andersen I.M., Kaczmarska J., McGrew S.G., Malow B.A. Melatonin for insomnia in children with autism spectrum disorders. J Child Neurol., 23: 482-5, 2008.

Angriman M., Caravale B., Novelli L., Ferri R., Bruni O. Sleep in Children with neuro developmental disabilities. Neuropediatrics, 46: 199-210, 2015.

Blumer J.L., Findling R.L., Shih W.J., Soubrane C., Reed M.D. Controlled clinical trial of zolpidem for the treatment of insomnia associated with attention-deficit/ hyperactivity disorder in children 6 to 17 years of age. Pediatrics, 123: e770-6, 2009.

Bootzin R.R. and Stevens S.J. Adolescents, substance abuse, and the treatment of insomnia and daytime sleepiness. Clin. Psychol. Rev., 25: 629-44, 2005.

Brescianini S., Volzone A., Fagnani C., Patriarca V., Grimaldi V., Lanni R., Serino L., Mastroiacovo P., Stazi M.A. Genetic and environmental factors shape infant sleep patterns: a study of 18-monthold twins. Pediatrics, 127: e1296-1302, 2011.

Bruni O., Addessi E., Angriman M., Riccioni A., Dosi., Ferri R. Toward a clinical and therapeutic classification of insomnia of childhood. Sleep Med., 14: e18-e92, 2013.

Bruni O., Alonso-Alconada D., Besag F., Biran V., Braam W., Cortese S., Moavero R., Parisi P., Smits M., Van der Heijden K., Curatolo P. Current role of melatonin in pediatric neurology: clinical recommendations. Eur. J. Paediatr. Neurol., 19: 122-133, 2015.

Bruni O., Angriman M., Luchetti A., Ferri R. Leg kicking and rubbing as a highly suggestive sign of pediatric RLS. Sleep Med., 16: 1576-1577, 2015.

Bruni O., Ferri R., Miano S., Verrillo E.L. -5-Hydroxytryptophan treatment of sleep terrors in children. Eur. J. Pediatr., 163: 402-407, 2004.

Bruni O., Violani C., Luchetti A., Miano S., Verrillo E., Di Brina C., Valente D. The Sleep Knowledge of Pediatricians and Child Neuropsychiatrists. Sleep and Hypn., 6: 130-138, 2004.

Bruni O., Ottaviano S., Guidetti V., Romoli M., Innocenzi M., Cortesi F., Giannotti F. The sleep disturbance scale for children (SDSC). Construction and validation of an instrument to evaluate sleep disturbance in childhood and adolescence. J. Sleep Res., 5: 251-261, 1996. 
Cortese S., Angriman M., Lecendreux M., Konofal E. Iron and attention deficit/hyperactivity disorder: What is the empirical evidence so far? A systematic review of the literature. Expert Rev. Neurother., 12: 1227-1240, 2012.

Cortese S., Konofal E., Bernardina B.D., Mouren M.C., Lecendreux M. Sleep disturbances and serum ferritin levels in children with attentiondeficit/ hyperactivity disorder. Eur. Child Adolesc. Psychiatry, 18: 393-399, 2009.

Earley C.J., Connor J.R., Beard J.L., Malecki E.A., Epstein D.K., Allen R.P. Abnormalities in CSF concentrations of ferritin and transferrin in restless legs syndrome. Neurology, 54: 1698-700, 2000.

France K.G., Blampied N.M., Wilkinson P. A multiple-baseline, double-blind evaluation of the effects of trimeprazine tartrate on infant sleep disturbance. Exp. Clin. Psychopharmacol., 7: 502-513, 1999.

Giannotti F., Cortesi F., Cerquiglini A., Bernabei P. An open-label study of controlled-release melatonin in treatment of sleep disorders in children with autism. J. Autism. Dev. Disord., 36: 741-752, 2006.

Greene G., Gregory A.M., Fone D., White J. Childhood sleeping difficulties and depression in adulthood: the 1970 British Cohort Study. J. Sleep Res., 24: 19-23, 2015.

Gregory A.M., Caspi A., Eley T.C., Moffitt T.E., O'Connor T.G. and Poulton R. Prospective longitudinal associations between persistent sleep problems in childhood and anxiety and depression disorders in adulthood. J. Abnorm. Child Psychol., 33: 157-163, 2005.

Gringras P. When to use drugs to help sleep. Arch. Dis. Child., 93: 976-981, 2008.

Hartmann E. and Spinweber C.L. Sleep induced by L-tryptophan. Effect of dosages within the normal dietary intake. J. Nerv. Ment. Dis., 167: 497-479, 1979.

Harvey C.J., Gehrman P., Espie C.A. Who is predisposed to insomnia: A review of familial aggregation, stress-reactivity, personality and coping style. Sleep Med. Rev., 18: 237-247, 2014.

Harvey A.G., Murray G., Chandler R.A., Soehner A. Sleep disturbance as transdiagnostic: consideration of neurobiological mechanisms. Clin. Psychol. Rev., 31: 225-235, 2011.

Honaker S.M. and Meltzer L.J. Bedtime problems and night wakings in young children: an update of the evidence. Paediatr. Respir. Rev., 15: 333-339, 2014.

Honaker S.M. and Meltzer L.J. Sleep in pediatric primary care: A review of the literature. Sleep Med. Rev., 25: 31-39, 2016.
Heussler H., Chan P., Price A.M., Waters K., Davey M.J., Hiscock H. Pharmacological and non-pharmacological management of sleep disturbance in children: an Australian Pediatric Research Network survey. Sleep Med., 14: 189-194, 2013.

Kordas K., Siegel E.H., Olney D.K., Katz J., Tielsch J.M., Chwaya H.M., Kariger P.K., Leclerq S.C., Khatry S.K., Stoltzfus R.J. Maternal reports of sleep in 6-18 month-old infants from Nepal and Zanzibar: association with iron deficiency anemia and stunting. Early Hum. Dev., 84: 389-398, 2008.

Kordas K., Siegel E.H., Olney D.K., Katz J., Tielsch J.M., Kariger P.K., Khalfan S.S., LeClerq S.C., Khatry S.K., Stoltzfus R.J. The effects of iron and/ or zinc supplementation on maternal reports of sleep in infants from Nepal and Zanzibar. J. Dev. Behav. Pediatr., 30: 131-9, 2009.

Körner E., Bertha G., Flooh E., Reinhart B., Wolf R., Lechner H. Sleep-inducing effect of L-tryptophane. Eur. Neurol., 25 Suppl 2: 75-81, 1986.

Kotagal S. Treatment of dyssomnias and parasomnias in childhood. Curr. Treat. Options Neurol., 14: 630-49, 2012.

Kotagal S. and Silber M.H. Childhood-onset restless legs syndrome. Ann. Neurol., 56: 803-7, 2004.

Kryger M.H., Otake K., Foerster J. Low body stores of iron and restless legs syndrome: a correctable cause of insomnia in adolescents and teenagers. Sleep Med., 3: 127-132, 2002.

LeBlanc M., Mérette C., Savard J., Ivers H., Baillargeon L., Morin C.M. Incidence and risk factors of insomnia in a population-based sample. Sleep, 32: 1027-37, 2009.

Lieberman H.R., Corkin S., Spring B.J., Wurtman R.J., Growdon J.H. The effects of dietary neurotransmitter precursors on human behavior. Am. J. Clin. Nutr., 42: 366-70, 1985.

McCarty D.E., Chesson A.L. Jr, Jain S.K., Marino A.A. The link between vitamin D metabolism and sleep medicine. Sleep Med. Rev., 18: 311-9, 2014.

Miano S. and Peraita-Abrados R. Pediatric Insomnia: clinical, diagnosis and treatment. Rev. Neurol., 58: 35-42, 2014.

Meltzer L.J. Clinical management of behavioral insomnia of childhood: treatment of bedtime problems and night wakings in young children. Behav. Sleep Med., 8: 172-189, 2010.

Meltzer L.J. and Mindell J.A. Systematic Review and Meta-Analysis of Behavioral Interventions for Pediatric Insomnia. J. Ped. Psychol., 39: 932-948, 2014. 
Merenstein D., Diener-West M., Halbower A.C., Krist A., Rubin H.R. The trial of infant response to diphenhydramine: the TIRED study--a randomized, controlled, patient-oriented trial. Arch. Pediatr. Adolesc. Med., 160: 707-12, 2006.

Mindell J.A., Emslie G., Blumer J., Genel M., Glaze D., Ivanenko A., Johnson K., Rosen C., Steinberg F., Roth T., Banas B. Pharmacologic management of insomnia in children and adolescents: consensus statement. Pediatrics, 117: e1223-32, 2006.

Mindell J.A., Kuhn B., Lewin D.S., Meltzer L.J., Sadeh A.; American Academy of Sleep Medicine. Behavioral treatment of bedtime problems and night wakings in infants and young children. Sleep, 29: 1263-1276, 2006.

Mindell J.A., Meltzer L.J., Carskadon M.A., Chervin R.D. Developmental aspects of sleep hygiene: findings from the 2004 National Sleep Foundation Sleep in America Poll. Sleep Med., 10: 771-779, 2009.

Mindell J., Owens J. A Clinical Guide to Pediatric Sleep. 2010, Philadelphia: Lipincott Williams \& Wilkins.

Mindell J.A., Sadeh A., Wiegand B., How T.H., Goh D.Y. Cross-cultural differences in infant and toddler sleep. Sleep Med., 11: 274-280, 2010.

Montanari G., Schiaulini P., Covre A., Steffan A., Furlanut M. Niaprazine vs chlordesmethyldiazepam in sleep disturbances in pediatric outpatients. Pharmacol Res., 25 Suppl 1: 83-84, 1992.

Monti J. Serotonin control of sleep-wake behavior. Sleep Med. Rev., 15: 269-281, 2011.

Morgenthaler T., Kramer M., Alessi C., Friedman L., Boehlecke B., Brown T., Coleman J., Kapur V., Lee-Chiong T., Owens J., Pancer J., Swick T. American Academy of Sleep Medicine. Practice parameters for the psychological and behavioral treatment of insomnia: an update. An american academy of sleep medicine report. Sleep, 29: 14151419, 2006.

Nguyen M., Tharani S., Rahmani M., Shapiro M. A review of the use of clonidine as a sleep aid in the child and adolescent population. Clin. Pediatr. Phila., 53: 211-216, 2014.

Owens J.; Adolescent Sleep Working Group; Committee on Adolescence. Insufficient sleep in adolescents and young adults: an update on causes and consequences. Pediatrics, 134: e921-32, 2014.

Owens J.A., Babcock D., Blumer J., Chervin R., Ferber R., Goetting M., Glaze D., Ivanenko A., Mindell J., Rappley M., Rosen C., Sheldon S. The use of pharmacotherapy in the treatment of pediat- ric insomnia in primary care: rational approaches. A consensus meeting summary. J. Clin. Sleep Med., 1: 49-59, 2005.

Owens J.A. Pharmacotherapy of pediatric insomnia. J. Am. Acad. Child. Adolesc. Psychiatry, 48: 99-107, 2009.

Owens J.A. and Mindell J.A. Pediatric insomnia. Pediatr. Clin. North Am., 58: 555-569, 2011.

Owens J.A., Babcock D., Blumer J., Chervin R., Ferber R., Goetting M., Glaze D., Ivanenko A., Mindell J., Rappley M., Rosen C., Sheldon $\mathrm{S}$. The use of pharmacotherapy in the treatment of pediatric insomnia in primary care; rational approaches. A consensus meeting summary. $J$. Clin. Sleep Med., 1: 49-59, 2005.

Ottaviano S., Giannotti F., Cortesi F. The effect of niaprazine on some common sleep disorders in children. A double-blind clinical trial by means of continuous home-videorecorded sleep. Childs Nerv. Syst., 7: 332-335, 1991.

Palagini L., Biber K., Riemann D. The genetics of insomnia--evidence for epigenetic mechanisms? Sleep Med. Rev., 18: 225-235, 2014.

Pelayo R. and Dubik M. Pediatric sleep pharmacology. Semin. Pediatr. Neurol., 15: 79-90, 2008.

Pelayo R. and Yuen K. Pediatric sleep pharmacology. Child. Adolesc. Psychiatr. Clin. N. Am., 21: 861-883, 2012.

Picchietti D.L., Rajendran R.R., Wilson M.P., Picchietti M.A. Pediatric restless legs syndrome and periodic limb movement disorder: parent-child pairs. Sleep Med., 10: 925-931, 2009.

Picchietti D.L. and Stevens H.E. Early manifestations of restless legs syndrome in childhood and adolescence. Sleep Med., 9: 770-781, 2008.

Reid G.J., Huntley E.D., Lewin, D.S. Insomnias of Childhood and Adolescence. Child. Adolesc. Psychiatric. Clin. N. Am., 18: 979-1000, 2009.

Reid M.J., Walter A.L., O'Leary S.G. Treatment of young children's bedtime refusal and nighttime wakings: a comparison of "standard" and graduated ignoring procedures. J. Abnorm. Child. Psychol., 27: 5-16, 1999.

Russo R.M., Gururaj V.J., Allen J.E. The effectiveness of diphenhydramine HCI in pediatric sleep disorders. J. Clin., Pharmacol., 16: 284-288, 1976.

Schneider-Helmert D. and Spinweber C.L. Evaluation of L-tryptophan for treatment of insomnia: a review. Psychopharmacology (Berl)., 89: 1-7, 1986.

Smits M.G., van Stel H.F., van der Heijden K., Meijer A.M., Coenen A.M., Kerkhof GA. Melatonin 
improves health status and sleep in children with idiopathic chronic sleep-onset insomnia: a randomized placebo-controlled trial. J. Am. Acad. Child. Adolesc. Psychiatry, 42: 1286-1293, 2003.

Thakkar M.M. Histamine in the regulation of wakefulness. Sleep Med. Rev., 15: 65-74, 2011.

Tilma J., Tilma K., Norregaard O., Ostergaard J.R. Early childhood-onset restless legs syndrome: symptoms and effect of oral iron treatment. Acta Padiatr., 102: e221-e226, 2013.

Touchette E., Dionne G., Forget-Dubois N., Petit D., Pérusse D., Falissard B., Tremblay R.E., Boivin M., Montplaisir J.Y. Genetic and environmental influences on daytime and nighttime sleep duration in early childhood. Pediatrics., 131: e1874-80, 2013.

Van der Heijden K.B., Smits M.G., Van Someren E.J., Gunning W.B. Idiopathic chronic sleep onset insomnia in attention-deficit/hyperactivity disorder: a circadian rhythm sleep disorder. Chronobiol. Int., 22: 559-570, 2005.
Van der Heijden K.B., Smits M.G., Van Someren E.J., Ridderinkhof K.R., Gunning W.B. Effect of melatonin on sleep, behavior, and cognition in ADHD and chronic sleep-onset insomnia. J. Am. Acad. Child. Adolesc. Psychiatry., 46: 233-241, 2007.

Van Geijlswijk I.M., van der Heijden K.B., Egberts A.C., Korzilius H.P., Smits M.G. Dose finding of melatonin for chronic idiopathic childhood sleep onset insomnia: an RCT. Psychopharmacology, 212: 379-391, 2010.

Wasdell M.B., Jan J.E., Bomben M.M., Freeman R.D., Rietveld W.J., Tai J., Hamilton D., Weiss M.D. A randomized, placebo-controlled trial of controlled release melatonin treatment of delayed sleep phase syndrome and impaired sleep maintenance in children with neurodevelopmental disabilities. J. Pineal Res., 44: 57-64, 2008.

Weiss M.D., Wasdell M.B., Bomben M.M., Rea K.J., Freeman R.D. Sleep hygiene and melatonin treatment for children and adolescents with ADHD and initial insomnia. J. Am. Acad. Child. Adolesc. Psychiatry., 45: 512-519, 2006. 